\title{
CONFIDENCE INTERVALS IN GENERAL REGRESSION MODELS THAT UTILISE UNCERTAIN PRIOR INFORMATION
}

\author{
NISHIKA RANATHUNGA (iD
}

(Received 28 December 2021; first published online 8 February 2022)

2020 Mathematics subject classification: primary 62F25; secondary 62J12, 65D30, 82M31.

Keywords and phrases: confidence interval, generalised linear model, Monte Carlo simulation, numerical integration.

We consider a general regression model without a scale parameter. We construct a confidence interval for a scalar parameter of interest that utilises the uncertain prior information that a distinct scalar parameter takes a specified value. This confidence interval has good coverage properties. It also has scaled expected length, where the scaling is with respect to the usual confidence interval, that is, (a) it is substantially less than 1 when the prior information is correct, (b) it has a maximum value that is not too large and (c) it is close to 1 when the data and prior information are highly discordant. We use the $\mathrm{R}$ package ciuupi, which computes a confidence interval that utilises uncertain prior information in a linear regression model with known error variance, to build this confidence interval. This work appears in [3] and has been submitted for publication.

We also solve the problem of numerically evaluating the expected value of a smooth bounded function of a chi-distributed random variable, divided by the square root of the number of degrees of freedom, using Mori's transformation followed by the trapezoidal rule, which is exponentially convergent for suitable integrands. This problem arises in simultaneous inference, selection and ranking of populations, the evaluation of multivariate $t$ probabilities and the assessment of the coverage and expected volume properties of nonstandard confidence regions. This work appears in [4].

We apply this solution in the new R package ciuupi2 [5] that computes the Kabaila and Giri [1] confidence interval which utilises uncertain prior information in a linear regression model with unknown error variance. Previous computations of this interval used MATLAB programs that were time-consuming to run. By writing these programs

Thesis submitted to La Trobe University in February 2021; degree approved on 9 June 2021; supervisor Paul Kabaila, co-supervisor David Farchione.

(C) The Author(s), 2022. Published by Cambridge University Press on behalf of Australian Mathematical Publishing Association Inc. 
in $\mathrm{R}$, the computation time is greatly reduced and they become freely available. We also assess a new definition of scaled expected length.

Finally, we compare the computations of the log-likelihood function for generalised linear mixed models using (a) adaptive Gauss-Hermite quadrature and (b) importance sampling, where both methods share the same initial step. This work appears in [2].

\section{References}

[1] P. Kabaila and K. Giri, 'Confidence intervals in regression utilizing prior information', J. Statist. Plann. Inference 139 (2009), 3419-3429.

[2] P. Kabaila and N. Ranathunga, 'On adaptive Gauss-Hermite quadrature for estimation in GLMM's', in: Statistics and Data Science (Research School on Statistics and Data Science, RSSDS 2019, Melbourne, Vic., Australia, July 24-26, 2019, Proceedings), Communications in Computer and Information Science, 1150 (ed. H. Nguyen) (Springer, Singapore, 2019), 130-139.

[3] P. Kabaila and N. Ranathunga, 'Confidence intervals in general regression models that utilize uncertain prior information', Preprint, 2020, arXiv:2009.07464.

[4] P. Kabaila and N. Ranathunga, 'Computation of the expected value of a chi-distributed random variable', Comput. Statist. 36 (2021), 313-332.

[5] N. Ranathunga and P. Kabaila, 'ciuupi2: Kabaila and Giri (2009) confidence interval', R package version 1.0.1. https://cran.r-project.org/web/packages/ciuupi2/.

NISHIKA RANATHUNGA, Department of Mathematics and Statistics,

La Trobe University, Bundoora, Victoria 3086, Australia

e-mail: kapuruge.n@wehi.edu.au 